\title{
A New Method for Identifying Otological Drill Milling Through Bone Wall
}

\author{
Xisheng $\mathrm{Li}^{1, a}$, Yunqing $\mathrm{Li}^{1, \mathrm{~b}}$, Guodong Feng ${ }^{2, \mathrm{c}}$, Zhiqiang $\mathrm{GaO}^{2, \mathrm{~d}}$ and Peng \\ Shen ${ }^{3, e}$ \\ ${ }^{1}$ School of Automation and Electrical Engineering, University of Science and Technology Beijing, \\ No.30, Xueyuan Road, Haidian District, Beijing 100083, China \\ ${ }^{2}$ Department of otolaryngology, Peking Union Medical College Hospital, Chinese Academy of \\ Medical Sciences and Peking Union Medical College, Beijing 100730, China \\ ${ }^{3}$ Department of Otolaryngology, Beijing Chuiyangliu Hospital Affiliated to Tsinghua University, \\ Beijing 100022, China
}

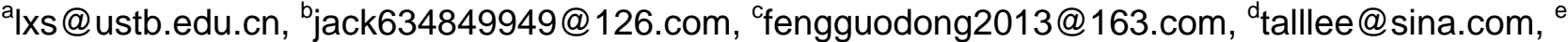
shen-peng2002@163.com

Keywords: Intelligent system, Otological drill, Multi-sensor information fusion

\begin{abstract}
Surgeons are trying to achieve higher qualities in minimally invasive operation. With the growth of modern technology, intelligent medical equipments are used to improve operation safety. Milling through the bone tissue wall is a common abnormal milling state in ear surgery, which will do harm to the patient's encephalic tissues. To avoid this kind of accident, some methods were researched by engineers or surgeons, and this paper presents a new method for identifying this kind of abnormal milling state. Five surgeons were invited to perform experiments on calvarian bones. The average recognition rate for otological drill milling through a bone tissue wall was 93\%, whereas only $2 \%$ of the normal millings were identified as abnormal states by error.
\end{abstract}

\section{Introduction}

With the improving living standards of human, the demand for better health care is growing [1]. As an important auxiliary tool in healing process, medical instruments plays an important role in treatments. The development of modern medicine has claimed higher and higher requirements for intelligent medical equipments. Surgical instruments are one kind of medical equipments. Over the past decades, surgical instruments have been continually improved, making operations much safer [2].

The otological drill is one of the fundamental tools in ear surgery. It is usually used for milling holes in the skull to remove diseased tissue or provide access for further intervention, including cochlear implantation [3]. It is controlled directly by the operating surgeon, and requires a high degree of coordination of hand and foot, with one hand controls its path and one foot controls its switch state. As an intracranial surgery of high risk, the surgical cavity for otology operation is small. The drill bit with high-speed rotation is easy to damage the patients' important intracranial organs [4].

Milling through the bone tissue wall is a common abnormal milling process. For intracranial operation, a hole on the bone has to be milled. When the bone is milled very thin, a slight touch may cause the high-speed rotating drill bit to break-through the bone wall and bring unexpected damage to the patient's tissues. It is difficult for surgeons to timely prevent the drill from penetrating the bone wall [5]. During the operation, surgeons have to estimate the thickness of the remaining bone covering over the patient's tissue. Therefore, if the otological drill is able to intelligently identify the abnormal milling state timely and make appropriate treatment automatically, the additional injury from the operation will be reduced.

Many efforts have been done by engineers to develop this kind of smart otological drill system. At the end of last century, F.R. Ong and Bouazza-Marouf had investigated a robust detection method for drill bit break-through when drilling into long bones using an automated drilling system [6]. They described a repeatable method of break-through detection based on a modified Kalman filter, which was applyed to the force difference between successive samples [7]. Wen-Yo Lee and Ching-Long 
Shih had described and a robotic bone drilling system for applications in orthopedic surgery in 2006. Their goal was to realize a three-axis robotic drilling system which can automatically stop drilling at the moment a drill breaks through bone [8]. Jaesung Hong and his partners have tried an image-guided surgical system for otologic surgery in 2008. With reliable hybrid registration, real-time patient movement compensation and virtual intraoperative computed tomography imaging have been originally proposed [9]. In 2014, Neal P. Dillon and his partners had developed a compact, bone-attached milling robot to mill away part of the temporal bone in mastoidectomy procedure. A positioning frame, containing fiducial markers and attachment points for the robot, is rigidly attached to the skull of the patient, and a CT scan is acquired. The target bone volume is manually segmented in the CT by the surgeon and automatically converted to a milling path and robot trajectory [10]. Some of the presented methods are based on automatic robots, by which surgeons cannot play their subjective initiative into the surgery. And the other methods may have a higher cost.

This paper presents a new method for identifying otological drill milling through the bone tissue wall. This method is not an expensive one, which can give surgeons the chance to play their subjective initiatives. Our tests show that the milling states of an otological drill can be identified precisely in real time. The average recognition rate for milling through bone wall was 93\%, whereas only $2 \%$ of normal millings were identified as abnormal milling states.

\section{Materials and Methods}

Sensor System. Many parameters are able to reflect the milling states, including motor current, milling force, torque, drill rotational speed [11]. Considering the correlation with milling process, two kinds of sensors, specifically a current sensor (CHB-25NP; Beijing SENSOR Electronics Company, Beijing, China) and a two-dimensional force sensor (Model RN20 strain gauge; Jinan Jinzhong Electronic Scale Corporation, Jinan, China), were installed on a modified otological drill (ZCW-1; 21st Institute, China’s Ministry of Machinery and Electronics, Shanghai, China).

The current sensor was used to measure the electromagnetic torque of a DC motor. The relationship between current $i$ and electromagnetic torque $T_{e}$ is as follows:

$$
T_{e}=C_{e} i \text {, }
$$

where $C_{e}$ is a constant. The measurement range of the current sensor is from 0 to $2.5 \mathrm{~A}$.

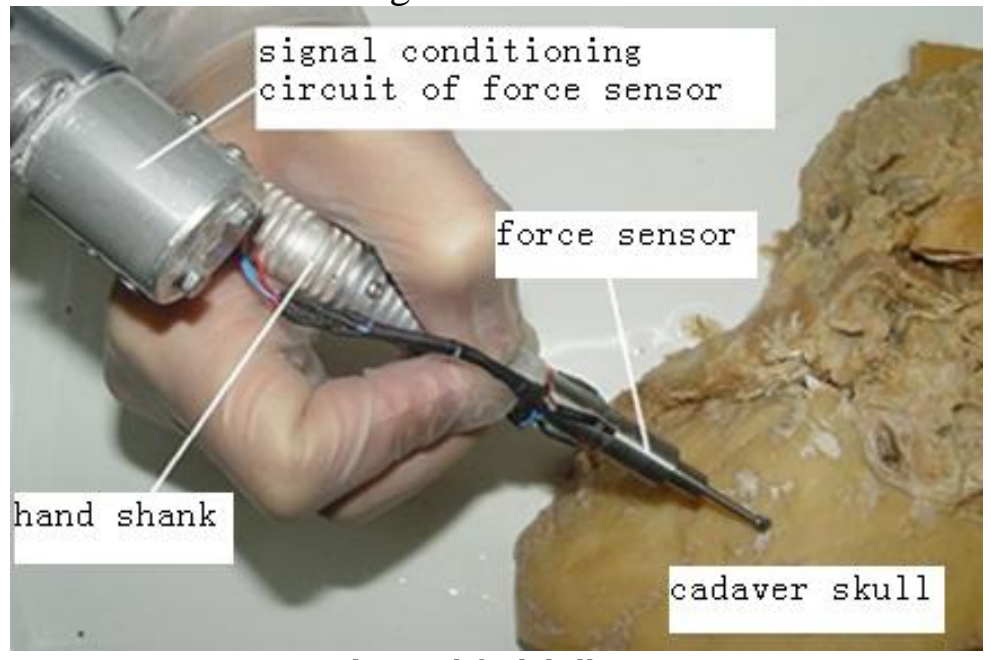

Fig. 1 The modified drill system

The force sensor was used to measure the normal cutting force at the milling surface. A sleeve was added in front of the drill handle. Through a sliding bearing installed in the front of the sleeve, the normal cutting force $F$ of the drill bit can be transferred to the sleeve. Four strain gauges were bonded onto the sleeve: These could measure the normal cutting force $F$ by measuring the elastic deformation occurred on the sleeve (Fig. 1). The force sensor could measure two orthogonal component forces $F_{X}$ and $F_{y}$. The normal cutting force $F$ is calculated as follows:

$$
F=\sqrt{F_{x}^{2}+F_{y}^{2}} \text {. }
$$


The measurement range of the force sensor is from $-5 \mathrm{~N}$ to $5 \mathrm{~N}$, with a resolution of $0.005 \mathrm{~N}$ and an accuracy of $0.05 \mathrm{~N}$.

The sample rate of each sensor signal channel is $1024 \mathrm{~Hz}$. To suppress sensor noise, an over-sampling method was used [12]. The grinding object used in the experiment was calvarian bone that had been fixed in formalin. All the experimental data are accessed and processed by a computer. Data Preprocessing. The original signal from the sensors has a lot of noises and interferences. After we use DB2 wavelet transform and reconstruction, we use average filter as the original data were over-sampled.

After the process above, the frequency of the input data has decreased from $1024 \mathrm{~Hz}$ to $64 \mathrm{~Hz}$. However, it's still not enough for recognizing only after removing noises, the values of the force sensors don't correspond to zero point when the drill is idling, since the force sensors' readings can be influenced by the gravity of the drill bit. During the surgeon's operation, the drilling angle is changing all the time. So the readings of each force sensor, $F_{x}$ or $F_{y}$, must be processed. The readings of force sensors before every drilling operation are changing, but the readings of current sensor are not, so we can judge whether the drill is idling by the readings of the current sensor, and calculate the average values of each force sensor before every drilling operation.

Mathematical Model of the DC Motor. According to the analyze to the DC motor [13], the change rules of the armature current and the motor speed can be described by the following equations:

$$
\begin{aligned}
& \frac{d i}{d t}=-\frac{r}{L} i-\frac{k}{L} \omega-\frac{1}{L} u \\
& \frac{d \omega}{d t}=-\frac{k}{J} i-\frac{B}{J} \omega-\frac{1}{J} T_{L}
\end{aligned}
$$

where $i$ is the armature current, $u$ is the armature voltage, $r 、 L$ are the equivalant resistance and equivalent inductance of the armature winding, $k$ is the torque constant determined by the permanent magnet and armature magnetic properties of the motor, $B$ is the coefficient of frictional resistance of the motor rotor, $T_{L}$ is the torque of the rotating load, $\omega$ is the rotor angular velocity, and $J$ is the total moment of inertia of the motor rotor and the drill bit.

The solutions of the differential eq. (3) were figured out as in eq. (4), where the $C_{1}$ and $C_{2}$ are constants.

$$
\begin{gathered}
i=-\frac{C_{1} L e^{-\frac{r}{L} t}+k \omega+u}{r} \\
\omega=-\frac{C_{2} J e^{-\frac{B}{J} t}+k i+T_{L}}{B}
\end{gathered}
$$

After eliminating the $\omega$, we got the expression of $i$ :

$$
i=\frac{-C_{1} L B e^{-\frac{r}{L} t}+C_{2} k J e^{-\frac{B}{J} t}+k T_{L}-u B}{r B-k^{2}} .
$$

To get the relationships between the armature current $i$ and the torque of the rotating load $T_{L}$, we write eq. (6) as below.

$$
i=C_{1} e^{C_{2} t}+C_{3} e^{C_{4} t}+C_{5} T_{L}+C_{6},
$$

where the $C_{1}$ to $C_{6}$ are constants, which differ from those in eq. (5). The first two terms in the right side of the eq. (6) are exponential terms, which will decrease with time. They are existent because of the rotary inertia of the drill.

If we use $i$ to express $T_{L}$, and ignore the exponential terms, the eq. (6) can be written as

$T_{L}=C_{k 1} i+C_{k 2}$,

where the $C_{k 1}$ and $C_{k 2}$ are constants.

Since the drill bit is doing relative slip movement with the bone surface during drilling, so the milling force can be recognized as sliding friction [14]. Its value $f$ approximately depends on the 
surface materials and the normal pressure $F_{N}$ [15]. The expression of $f$ is

$$
f=\mu F_{N}=\mu \sqrt{\left(F_{a} \sin \theta\right)^{2}+\left(F_{j} \cos \theta\right)^{2}},
$$

where the $\mu$ is the coefficient of sliding friction, $\theta$ is the angle between the axis and the grinding surface, $F_{a}$ is the axial force, and $F_{j}$ is the radial force.

During normal milling operation, the axial force is usually much smaller than the radial force, so we think that the $T_{L}$ during normal milling is in proportion to the radial force $F_{j}$, this relationship can be described in the following equation:

$$
T_{L}=k_{t} F_{j} \text {, }
$$

where $k_{t}$ is milling resistance coefficient that depends on the physical property of the grinding object. The higher $k_{t}$ is, the harder the object could be. The value of $k_{t}$ can be changing, while the value of $F_{j}$ can be measure by the current and $k_{t}$. According to eqs. (7) and (9), we got the equation below:

$$
F_{j}=\frac{C_{k 1} i+C_{k 2}}{k_{t}} .
$$

Since every term in the right side of eq. (10) has their coefficient, we set the value of $k_{t}$ to be 1 during normal milling, so that it will be easier to compare its values in normal milling and abnormal milling. Matlab was used to fit these two coefficients, $C_{k 1}$ and $C_{k 2}$, through the current and force data during normal milling.

The Phase Difference and Its Influence. Now we use the coefficients fitted by the data collected from normal milling, $C_{k 1}$ and $C_{k 2}$ in eq. (10), to calculate the curve of $k_{t}$. The value of $k_{t}$ is expected to be around 1, and its expression is shown in the following formula:

$$
k_{t}=\frac{C_{k 1} i+C_{k 2}}{F_{j}} \text {. }
$$

The curve of $k_{t}$ during normal milling is shown in Fig. 2 . We can easily find that when $F_{j}$ increases rapidly, $k_{t}$ will suddenly decrease, and when $F_{j}$ decreases rapidly, $k_{t}$ will suddenly increase.
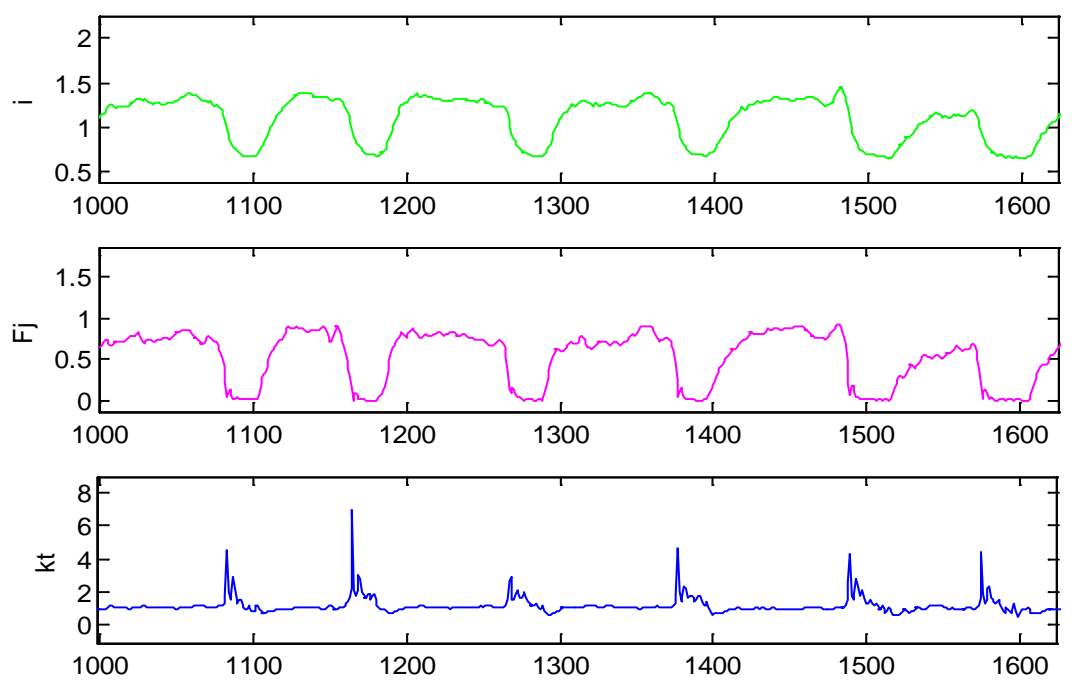

Fig. 2 The comparison of $i, F_{j}$ and $k_{t}$ during normal milling

Cao Tianyang from University of Science and Technology Beijing found that, there's phase difference between the force signal and current signal [16]. This phenomenon can be explained by eq. (4) and the curve of $k_{t}$ during normal milling (Fig. 2). There's an exponential term about time in the rotate speed expression of eq. (4), and the variation amplitude with time of this exponential term depends on $J$ and $B$, the moment of inertia and the coefficient of frictional resistance of the motor rotor.

From Fig. 2 we can find that, when the drill's status changes from idling to milling, the value of $k_{t}$ will suddenly decrease. This is because when the resistance to the drill bit increases rapidly, and the 
rotate speed decreases quickly, some of the rotational kinetic energy of the drill will help the electric energy to overcome the resistance, so the current increases relatively slow. As the result, the value of $k_{t}$ has a process of rapid decline. Likewise, the value of $k_{t}$ will suddenly increase while the drill's status changes from milling to idling.

When the drill bit is milling through the bone wall, it will get extra resistance besides milling force. At this time, the delay of the current signal comparing to the force signal seems to decrease, and even reverse [16]. Cao didn't tell why this happens, and he used moving average filter to eliminate the influence of the phase difference, regarding it as a measurement error. Actually, the phase difference should be constant if the DC motor was given. We can treat it as systematic error, so that we can take more advantage of the information from sensors [17].

Eliminating the Influence of Phase Difference. From the discussion above, we know that $k_{t}$ is a key parameter in distinguishing whether the state is abnormal, which can be influenced by the phase difference between the current signal and the force signal.

To eliminating the influence of phase difference, we do differentiations to both sides of eq. (6):

$$
\frac{d i}{d t}=C_{1} C_{2} e^{C_{2} t}+C_{3} C_{4} e^{C_{4} t}+C_{5} \frac{d T_{L}}{d t} .
$$

According to eq. (12), the change rate of current $i$ and that of $T_{L}$ only differs by two exponential terms. And eq. (5) shows that these two exponential terms both deceases with time. That's to say, if we shift the time axis of current $i$ to an earlier time, the change rate of current $i$ will be roughly in proportion to that of $T_{L}$.

Now the question is, how long time we shift earlier the time axis of current $i$ will lead to the approximately proportional relationship? We think it will happen when the variance of the $k_{t}$ array get the minimum value. And this equals to when the following function $f(m)$ with the independent variable $m$ have the minimum value:

$$
\begin{aligned}
& f(m)=\operatorname{Var}\left(k_{t}(n, m)\right) \\
& k_{t}(n, m)=\frac{C_{k 1, m} i_{n}+C_{k 2, m}}{F_{j, n-m}} .
\end{aligned}
$$

Here $k_{t}(n, m)$ is a two-dimensional matrix, where $n$ is the point in the time array, whose value is a positive integer, and $m$ is the number of time points that we shift earlier the time axis of current $i$, or we shift later the time axis of $F_{j} . i_{n}$ is the value of current sensor in time point $n$, and $F_{j, n-m}$ is the resultant of $F_{X}$ and $F_{y}$ in time point $n-m . C_{k 1, m}$ and $C_{k 2, m}$ are the the coefficients fitted by the shifted data, $i_{n}, i_{n+2}, i_{n+2} \ldots$ and $F_{j, n-m}, F_{j, n-m+1}, F_{j, n-m+2} \ldots$ which are collected from normal milling.

As the frequency of the data is $64 \mathrm{~Hz}$, the time between two nearest time points is $1 / 64 \mathrm{~s}$.

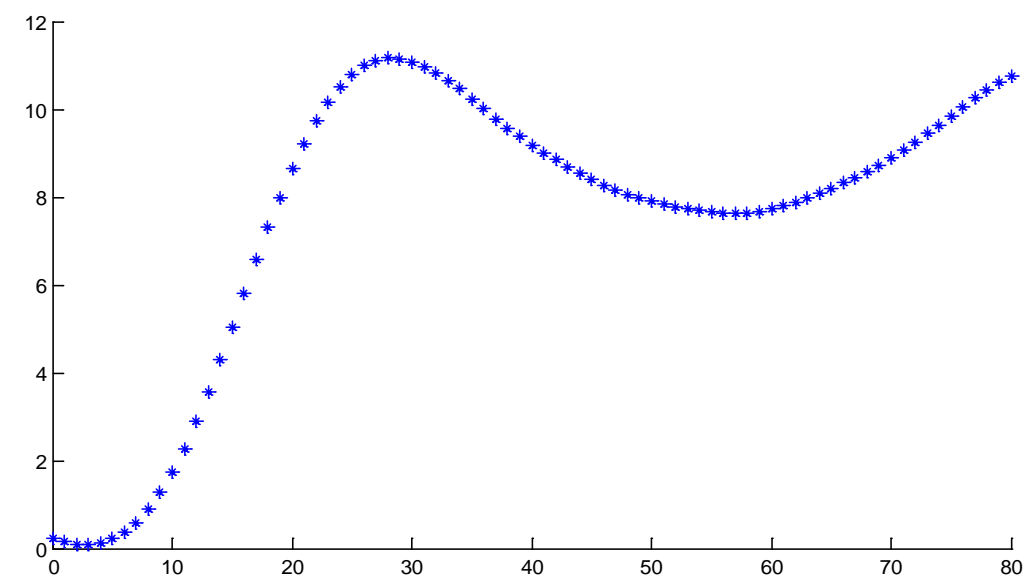

Fig. 3 The relationship between $f(m)$ and $m$, where the horizontal axis represents $m$ and the vertical represents

$$
f(m)
$$

When $m$ values from 0 to 80, integer only, the functional relationship between $f(m)$ and $m$ is shown in Fig. 3. When $m$ values $2, f(m)$ will get the minimum value. It's about 0.03 s that we shifted earlier 
the time axis of current $i$.

Although when $m$ values 2, $f(m)$ has a minimum value if $m$ must be an integer, what if $m$ can be a decimal number? To get a more accurate $m$, we do linear interpolation to the force data that are used to fit coefficients. From the results above, we know the value of $m$ we wanted is between 2 and 3 . So the force data that are shifted earlier by time point number $m$ can be calculated by the following expression:

$$
F_{j(n-m)}=F_{j(n-2)} \cdot(3-m)+F_{j(n-3)} \cdot(m-2), 2<m<3 .
$$

We set the interval of interpolation to 0.02 , and then calculated the fifty-one values of $f(m)$. When $m$ values 2.42, $f(m)$ gets the minimum value. We compared the curve of $k_{t}$ when $m$ values 2 and 2.42 (Fig. 4), and found that the fluctuation of $k_{t}$ when $m$ valued 2.42 was smaller than $m$ valued 2.

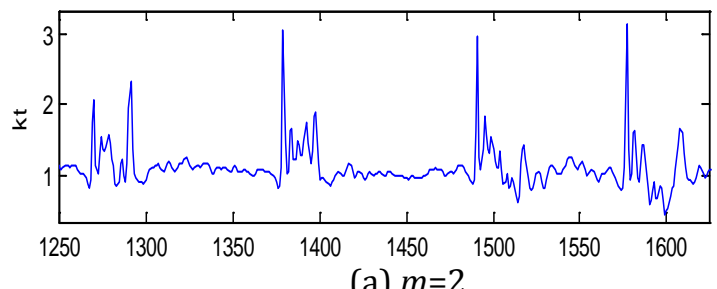

(a) $m=2$

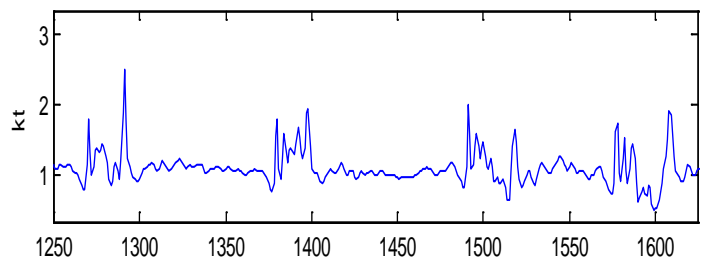

(b) $m=2.42$

Fig. 4 Comparison of the curve of $k_{t}$ when $m$ values 2 and 2.42

The value of $k_{t}$ is unstable when the current decreases rapidly. To eliminate this interference, we do a revise to the calculation of $k_{t}$ to make it more smooth and steady when the current decreases rapidly. At the same time, we add a number to the denominator of the expression of $k_{t}$ to make it steadier during idling. So $k_{c}$, the correction value of $k_{t}$, can be calculated by the following equation:

$$
k_{c, n}=\frac{C_{k 1} i+C_{k 2}+C_{k 3}\left(i_{n}-i_{n-1}\right)}{F_{j, n}+C_{k 4}},
$$

where $C_{k 1}=1.3240, C_{k 2}=-0.8763, C_{k 3}=1, C_{k 4}=0.2 . k_{c, n}, i_{n}$ and $F_{j, n}$ are the values of $k_{c}, i$ and $F_{j}$ in moment $n$.

Since the coefficients, $C_{k 1}$ and $C_{k 2}$, are both determined by the properties of the DC motor, but not the grinding objects, we use them as standard coefficients in the next.

Recognition Algorithm. According to doctors' operating custom and experimental data, the change rate of the current during normal milling is usually small, not exceeding 5A/s. But when it's drilling through the bone wall, the change rate can reach 20A/s. This is because when the milling through process is happening, the edge of the bone hole has a larger resisting moment to the concave bit.

We should set thresholds for the current change rate to judge whether it's drilling through the bone wall. Here the thresholds, include upper threshold and lower threshold, are determined by $\overline{\Delta i}$, the average increment rate of the current signal during normal milling. Only when the increment rate of the current signal is positive we use it to calculate the average value.

Likewise, the thresholds for the increment rate of $k_{c}$, include upper threshold and lower threshold, are determined by $\overline{\Delta k_{c}}$, the average increment rate of $k_{c}$ during normal milling. And also, only when the increment rate of $k_{c}$ is positive we use it to calculate the average value.

Both $\overline{\Delta i}$ and $\overline{\Delta k_{c}}$ should be continuously revised during milling operation.

The final judgment basis for drilling through bone wall can be realized by a piecewise function. We use the function value $P_{t h, n}$ to represent the chance of milling through in time point $n$.

The value of $P_{t h, n}$ is the summation of two parts, one determined by the increment rate of $i$, and the other by increment rate of $k_{c}$. both parts have equal weighting. For either of the two increment rate, if it's smaller than its lower threshold, then it will contribute zero to the value of $P_{t h, n}$; if it's between the lower and upper thresholds, its contribution to $P_{t h, n}$ will changes from 0 to 0.5 linearly; if it's larger than the upper threshold, it will increase $P_{t h, n}$ 's value by 0.5 . That's to say, if both the increment rates of $i$ and $k_{c}$ reaches their upper thresholds, $P_{t h, n}$ will value 1 .

The expression of $P_{t h, n}$ can be described by eq. (16): 


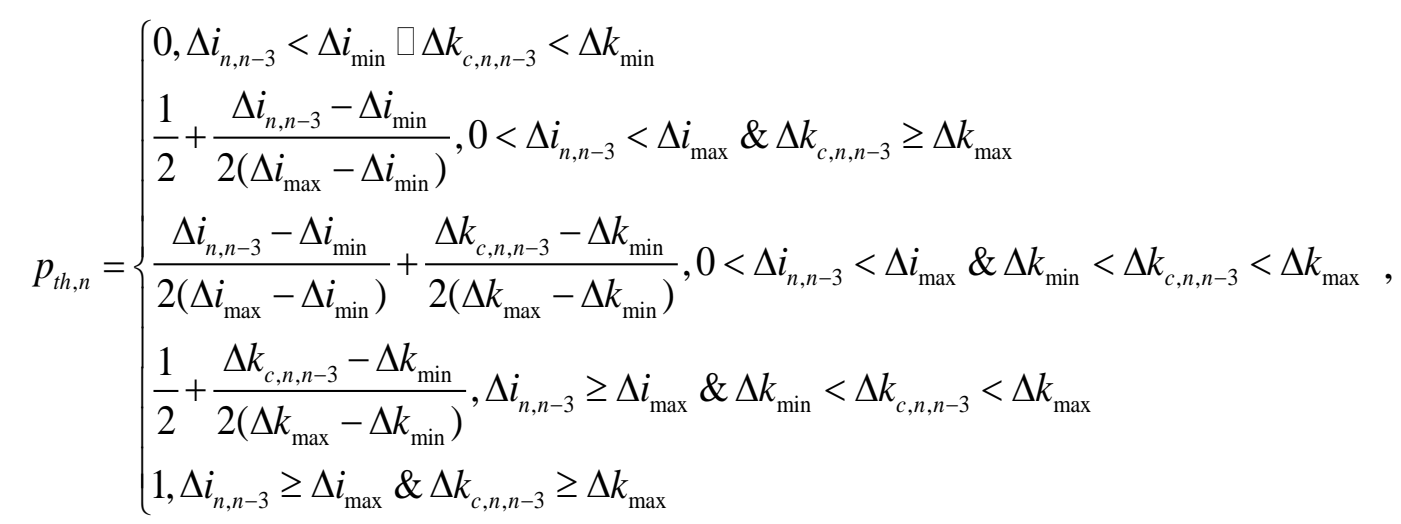

where $\Delta i_{\min }, \Delta i_{\max }, \Delta k_{\min }$ and $\Delta k_{\max }$ are all thresholds determined by $\overline{\Delta i}$ and $\overline{\Delta k_{c}}$. And $\Delta i_{n, n-3}=i_{n}-i_{n-3}, \Delta k_{c, n, n-3}=k_{c, n}-k_{c, n-3}$, where $i_{n}$ and $k_{c, n}$ represent the value of $i$ and $k_{c}$ in time point $n$.

If $P_{t h, n}>0.5$, we think that milling through bone wall happens.

\section{Result and Discussion}

We have done tests to prove the effectiveness of the recognition algorithms we presented above, using the experimental data collected from five surgeons.

During five hundred times' experiments of milling through bone tissue wall of five doctors, totally 465 of them were recognized. The recognition rate is $93 \%$. When we used the judgment function for milling through bone wall in 250 times' normal millings, only five times in total were recognized as milling through. The error rate was $2 \%$. As the result, we can say that the recognition algorithm for milling through bone wall is effective. The recognition of milling through bone tissue wall is clearly shown in Fig. 5.

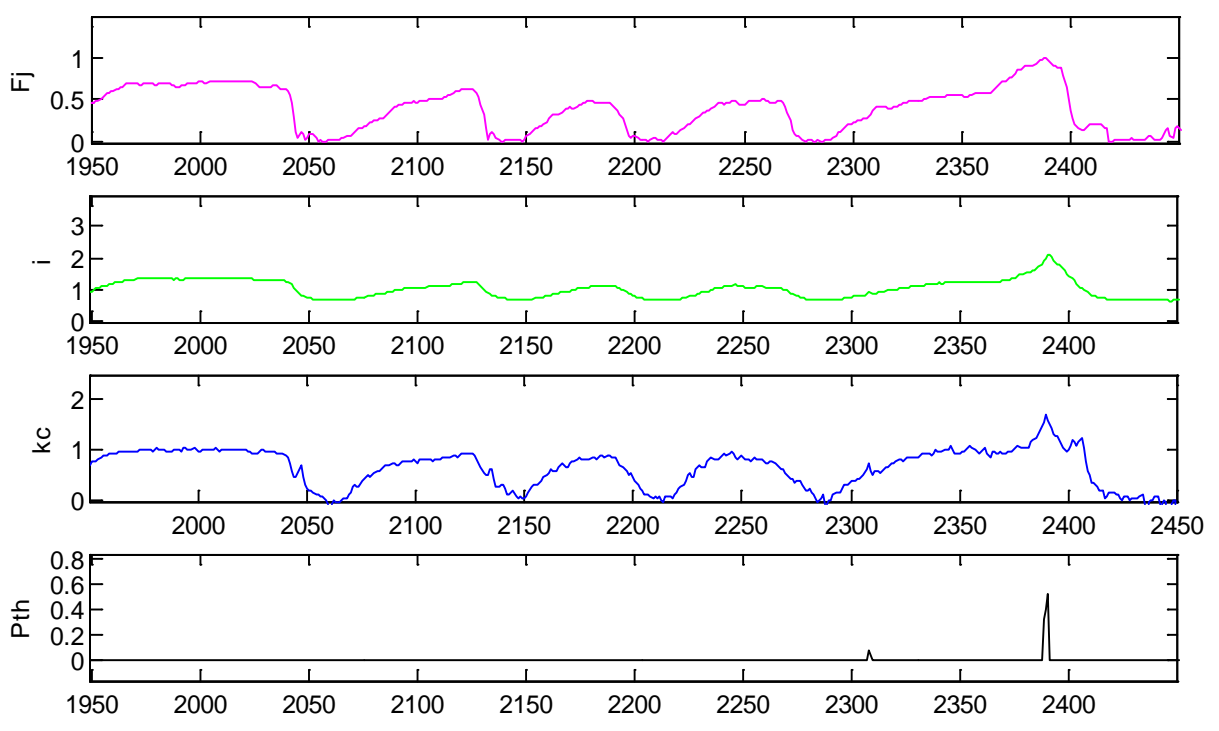

Fig. 5 The recognition of milling through bone tissue wall

Our final discriminant for milling through contains two indices, the change rate of $k_{c}$ and that of current signal. During normal milling, if the current signal increases rapidly only because the surgeon presses hard suddenly, the value of $k_{c}$ will not undergo a sudden change. However, when the drill bit is milling through the bone wall, there will be not only a sudden increment of current signal, but also an obvious increment of $k_{c}$ because of the increased resistance.

Surgeons usually operate carefully, so it seldom happens that current signal increases suddenly during normal milling. But to ensure the accuracy, $k_{c}$ is calculated and plays a complementary role. 


\section{Conclusion}

This paper presents a new method to identify the milling states of an otological drill. This multi-sensor based otological drill system can identify the early stage of milling through a bone tissue wall timely and accurately, with few false alarms during normal milling process. The proposed identification method can adapt to different surgeons and assist them to finish otological operations with less unexpected injuries to patients.

\section{Acknowledgment}

This work was funded under the National Key Technology R\&D Program (No.2012BAI12B01) of China during the 12th Five-year Plan Period, and the Major Construction Project of Beijing Personnel Training Co-Construction Project (The construction of strategic emerging industries related major).

\section{References}

[1] C. de Blok, K. Luijkx, B. Meijboom, J. Schols: BMC Health Services Research. Vol. 10 (2010), p.278.

[2] M. Sheedy, M. Bergin, G. Wylie, P. Ross, R. Dove, P. Bird: Australasian Physical \& Engineering Sciences in Medicine. Vol. 35 (2012), p.503.

[3] JD. Green, C. Shelyon, DE. Brachmann: Laryngoscope. Vol. 104 (1994), p.922.

[4] P. Shen, G.D. Feng, T.Y. Cao, Z.Q. Gao, X.S. Li: Int J Med Robotics Comput Assist Surg. Vol. 5(2009), p.284.

[5] T.Y. Cao, X.S. Li, Z.Q. Gao, G.D. Feng, P. Shen: Int J Med Robotics Comput Assist Surg. Vol. 7 (2011), p.148.

[6] F.R. Ong, K. Bouazza-Marouf: Proceedings of the Institution of Mechanical Engineers, Part H: Journal of Engineering in Medicine. Vol. 212(1998), p.209.

[7] F.R. Ong, K. Bouazza-Marouf: Mechatronics. Vol. 9 (1999), p.565.

[8] W.Y. Lee, C.L. Shih: Mechatronics. Vol. 16 (2006), p.73.

[9] J. Hong, N. Matsumoto, R. Ouchida, S. Komune, M. Hashizume: IEEE Transactions on Biomedical Engineering. Vol. 56 (2009), p.426.

[10]N. P. Dillon, R. Balachandran, A. M. dit Falisse, G. B. Wanna, R. F. Labadie, T. J. Withrow, J. M.Fitzpatrick, R. J. Webster: Medical Imaging: Image-Guided Procedures, Robotic Interventions, and Modeling. Vol. 9036 (2014), p.903614.

[11]X.S. Li, Y.M. Xie, Z.Q. Gao, G.D. Feng: Applied Mechanics and Materials. Vol.571-572 (2014), p.331.

[12]EA. Sandler, DA. Gusev, GY. Milman, ML. Podolsky: Signal Processing. Vol. 59 (1997), p.305.

[13]S.A. Nasar, L.E. Unnewehr: Schaum's Outline of Theory and Problems of Electric Machines and Electromechanics. 2nd ed. (McGraw-Hill, USA 1997).

[14]Z.F. Li ,H.B. Yang, C.T. Xu, X.Y. Chen, X.X. Liu: Natural Gas Industry. Vol. 33 (2013), No.12, p.1.

[15]I.S. Kang, J.S. Kimb, J.H. Kim, M.C. Kang, Y.W. Seo: Journal of Materials Processing Technology. Vol. 187 (2007), p.250.

[16]T.Y. Cao: Study on Multi-sensor Information Fusion Monitor System of Otological Drill. dissertation of University of Science and Technology Beijing, Beijing, China (2012).

[17]Q. Song, Y. He, W. Xiong: Journal of Southwest Jiaotong University, Vol.46 (2011), p.681. 